\title{
HIGH-FIDELITY AND LOW-ORDER MODELING OF A TRAVELING-WAVE THERMOACOUSTIC ENGINE
}

\author{
Carlo Scalo $^{1 *}$, Sanjiva K. Lele ${ }^{2}$, Lambertus Hesselink ${ }^{3}$ \\ ${ }^{1}$ School of Mechanical Eng. and Aeronautics and Astronautics, Purdue University, IN, 47907, USA \\ *Corresponding author's e-mail: scalo@purdue.edu (current affiliation) \\ ${ }^{2}$ Dept. of Aeronautics and Astronautics and Mechanical Eng., Stanford University, CA, 94305, USA \\ ${ }^{3}$ Dept. of Aeronautics and Astronautics and Electrical Eng., Stanford University, CA, 94305, USA
}

Keywords: thermoacoustic instability, streaming, traveling-wave, thermal-buffer tube, Stirling cycle, Lagrangian dynamics

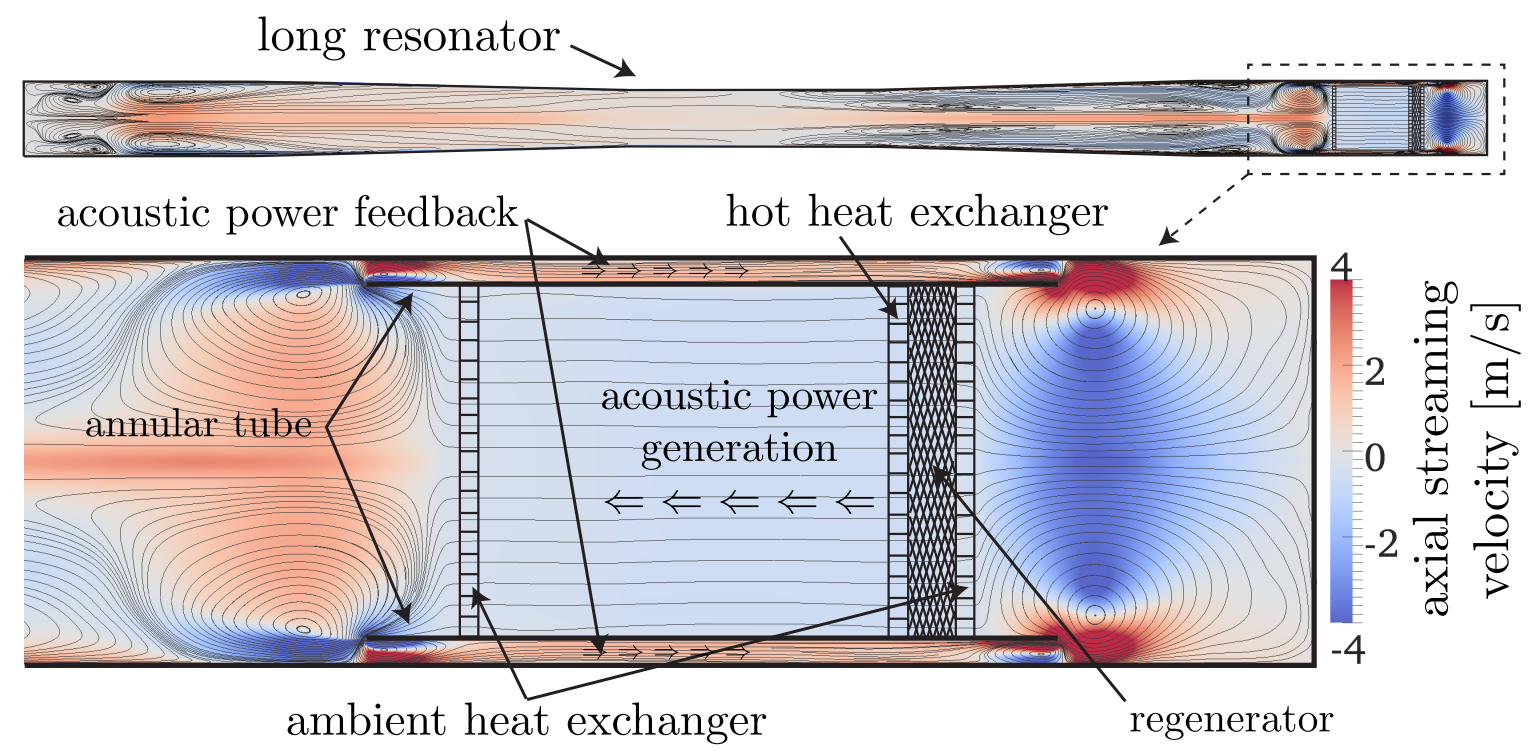

Figure 1: Contours of axial component of time-averaged streaming velocity $\left\langle\tilde{u}_{x}\right\rangle$ for $T_{h}=500 \mathrm{~K}$. Full-scale visualization (a) and zoom on the right end $(b)$.

Inspired by the work of Lycklama à Nijeholt et al. (2005) [1], Scalo et al., (2015) [2] have devised a simple traveling-wave TAE model that could serve as a benchmark case for high-fidelity numerical simulations of similar devices. We have extended such setup to three-dimensions and introduced a second ambient heat-exchanger to achieve a limit cycle, modeling typical fluid dynamic conditions found in thermal buffer tubes. While the simplicity of the adopted REG/HX heat-transfer and drag models allows for the straightforward development of companion linear and nonlinear models (necessary for verification of the Navier-Stokes calculations), other important processes typically occurring in REG/HX are, inevitably, not directly simulated.

The time integration is carried out from initial quiescent conditions to the limit cycle. It is shown that the mechanisms responsible for the acoustic energy generation and propagation in the system during the start-up phase can be explained with linear acoustics, despite the high amplitude ( $\sim 1 \%$ of the mean) of the initial perturbation resulting from the activation of the source terms modeling the heat transfer. An analytical linear Lagrangian model shows that the thermoacoustic instability occurring in the regenerator/heat-exchanger (REG/HX) unit intensifies plane waves traveling in the direction of the imposed temperature gradient via a process resembling a thermodynamic Stirling cycle. The result is the establishment of a network of selfamplifying traveling waves looping around the REG/HX unit. A system-wide linear stability 
model based on Rott's theory accurately predicts the frequency of the (only) unstable mode as well as the critical temperature ratio, despite not accounting for viscous and other nonlinear losses. The dependency of growth rates and limit-cycle pressure amplitudes on the temperature ratio are shown to be consistent with a supercritical Hopf bifurcation model. No evidence has been found to support subcritical or non-modal instability arguments.

At the limit cycle acoustic amplitudes exceed $+170 \mathrm{~dB}$ and nonlinear effects dominate the flow field in the form of transitional turbulence and acoustic streaming. The latter is the occurrence of a quasi-steady flow evolving over time scales much longer than the period of the waves inducing it. The data from the full three-dimensional simulations has allowed to identify the governing processes driving the streaming flow, which are viscous wave amplitude decay in the feedback inertance, periodic vortex ring roll-up and break-up around the sharp edges of the annular tube, and near-wall acoustic shear-stresses in the variable-area resonator.

An axially-symmetric numerical model based on Stokes-streamfunction formulation has been adopted to directly simulate the streaming flow as the solution of the incompressible NavierStokes equations driven by the divergence of the wave-induced Reynolds stresses extracted from the fully compressible three-dimensional calculations. The model correctly reproduces the streaming flow patterns and, in spite of the strong assumptions made and numerical issues associate with geometric singularities, it correctly predicts the intensity of the Gedeon streaming. The latter is responsible for the decrease of the engine's efficiency as the drive ratio is increased, and a robust parametrization for it is warranted. The investigation of the scaling of nonlinear fluxes reveals the importance of prior knowledge of the critical temperature ratio, which may not be straightforwardly achieved by simply relying on linear theory, for more complex systems. More details can be found in Scalo et al., (2015) [2].
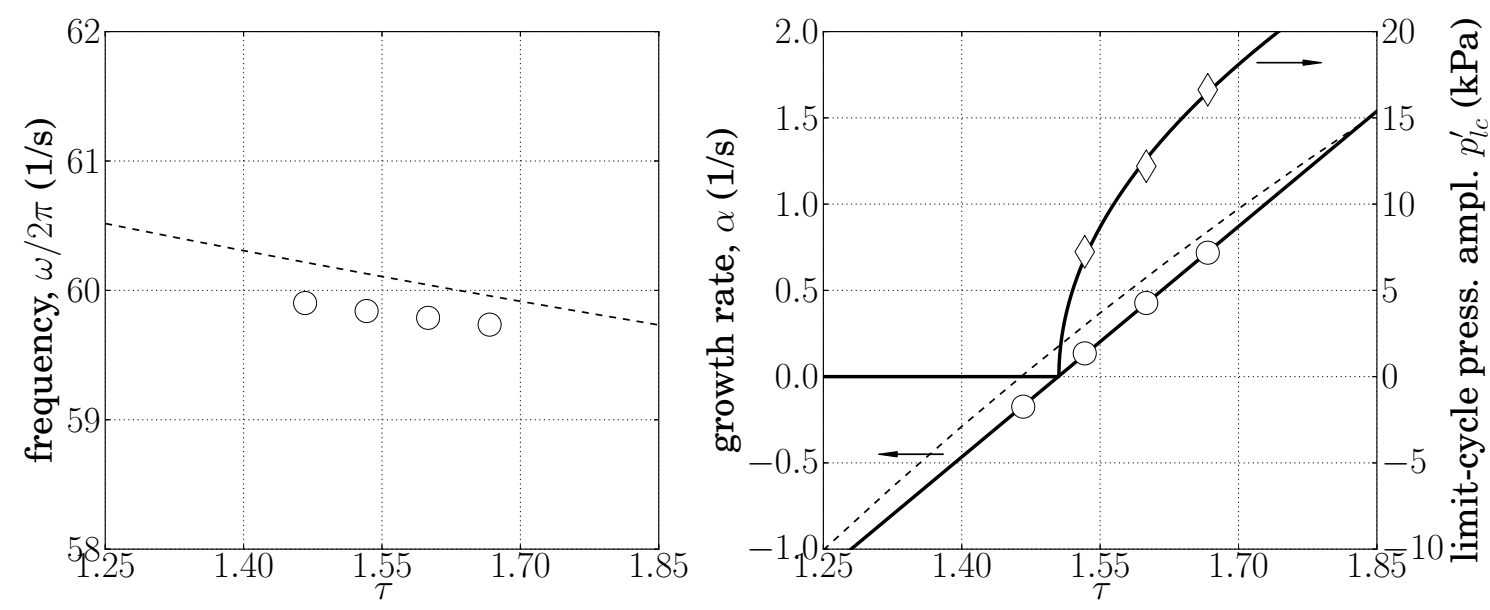

Figure 2: Frequency, $\omega / 2 \pi(a)$, growth rate, $\alpha$, and limit-cycle pressure amplitude $p_{l c}^{\prime}(b)$ versus hot-to-cold temperature ratio, $\tau=T_{h} / T_{c}$. Linear stability model (---), numerical simulations (symbols) with corresponding fitting ( - ) yielding critical temperature ratio $\tau_{c r}=1.505$ and $\left.p_{l_{c}}^{\prime}\right|_{\delta \tau=1}=41,000 \mathrm{~Pa}$.

\section{References}

[1] Lycklama à Nijeholt, J., Tijani, M., and Spoelstra, S. "Simulation of a traveling-wave thermoacoustic engine using computational fluid dynamics". J. Acoust. Soc. Am., 118, (2005), 2265-2270.

[2] Scalo, C., Lele, S. K., and Hesselink, L. "Linear and Nonlinear Modeling of a Theoretical Traveling-Wave Thermoacoustic Heat Engine". J. Fuid Mech., 766, (2015), 368 - 404. 